\title{
Effects of Sheds on Antioxidative Substances of Ziziphus Jujuba 'Zhanshanmizao'
}

\author{
Xi Wang ${ }^{1, a}$, Meihong Guo ${ }^{1, b}$, Yan Zhou ${ }^{1, c}$ and Qunxian Deng ${ }^{1, d *}$ \\ ${ }^{1}$ College of Horticulture, Sichuan Agricultural University, Chengdu, Sichuan, China \\ a1030706674@qq.com, blinxiao0617.h@qq.com, 'zhouyan6213500@qq.com, \\ ${ }^{d} 1324856299 q q . c 0 m$ \\ ${ }^{*}$ Corresponding author.
}

\begin{abstract}
Keywords: Ziziphus jujuba 'Zhanshanmizao'; shed; antioxidant; bud dormancy
Abstract. In this experiment, the 7-year-old Ziziphus jujuba 'Zhanshanmizao' tree with the same tree potential was used as the test material, and different time (December 5 th, 2016 and January 16 th, 2017) was selected to be treated with their sheds and exposed to uncovered open fields for comparison. The contents of antioxidative substances malonyldialdehyde (MDA), superoxide dismutase (SOD), peroxidase (POD), and $\mathrm{O}_{2}^{-} \cdot$ in the shoots of jujube trees were measured at each treatment level, the mechanism of the dormancy release of $Z$. jujuba 'Zhanshanmizao' was explored. The results showed that (1) the two treaments of jujube trees were relieved in January 17 th and around January 17 th. The contrast were relieved around February 14 th, and the two treatments of jujube trees relieved the dormancy about $30 \mathrm{~d}$ in advance. Comparing the effect of the two treatments, shed 2 was better than shed 1. (2) in the process of dormancy release, the treatments with shed significantly increased the activities of POD and SOD. (3) the contents of MDA and $\mathrm{O}_{2}{ }^{-}$. in $Z$. jujuba 'Zhanshanmizao' shoots with shed were less than contrast.
\end{abstract}

\section{Introduction}

Ziziphus jujuba 'Zhanshanmizao' was a plant of the Zizyphus. Mill of Rhanmaceae.R. Br. It is one of the jujube fresh foods with excellent flavor. It is rich in many vitamins and amino acids needed by the body, as well as rich mineral elements and medicinal substances. It has a very high therapeutic value and a variety of health care functions. It is known as the "nourishing and strong body health care product" [1]. It is an excellent fresh edible jujube with local characteristics that has been well received by the market in recent years [2].

Since 1980s, the cultivation of fruit trees has risen in China. Use of solar greenhouses, plastic greenhouses and other protective facilities to change or control the environmental conditions of fruit tree growth and development, artificially create a microclimate environment suitable for the growth and development of fruit trees, and achieve the artificial regulation of fruit tree production mode in order to achieve off-season supply, anti-seasonal sales [3]. At present, due to the limitation of the required amount of cooling, the fruit ripening period of most fruit trees such as peaches, apricots, pears, and grapes is first advanced to March, but still cannot be listed before the Spring Festival, thus restricting the annual supply of fresh fruit trees (except strawberry) [4]. In recent years, people have gradually paid attention to the study of bud dormancy mechanism and biochemistry, and research on jujube trees [5] has gradually increased.

The mature period of Z. jujuba 'Zhanshanmizao' is generally from late August to early September. Since the Z. Jujuba 'Zhanshanmizao' is relatively concentrated on the market, the fresh fruit is not resistant to storage, and the storage time is about 4 to 7 days at room temperature, resulting in a short shelf life, often resulting in slow sales, low prices, and even fruit rot, resulting in great economic losses. Therefore, we should carry out the experiment of shed so as to increase the maturity of $Z$. jujuba 'Zhanshanmizao' and increase the income of jujube farmers. By testing the physiological and biochemical changes in the natural dormancy of Z. jujuba 'Zhanshanmizao', we should make clear the dormancy characteristics of it, and explore the effective time for early maturity of it in different periods, and make clear the mechanism of breaking up the dormancy of it. The regulation of sleep 
provides a theoretical basis and provides technical guidance for regulating the maturity of $Z$. jujuba 'Zhanshanmizao' industry.

\section{Materials and Methods}

Materials. The experiment was carried out from October 2016 to September 2017 in Yonglian Village, Yongxin Town, Santai County, Mianyang City, Sichuan Province. The experimental material was a 7-year-old Z. jujuba 'Zhanshanmizao' (tiller propagation seedling). The row spacing is $2 \mathrm{~m} \times 3$ $\mathrm{m}$. The orchard is a hilly platform with yellow-purple soil.

December 5 th, 2016 (shed 1) and January 16 th, 2017 (shed 2) were selected to be treated with their sheds and exposed to uncovered open fields for comparison. In the single plant plot, each treatment was repeated 4 times, and a total of 12 jujube trees were used for the experiment.

The shed is made of steel frame, $4 \mathrm{~m}$ wide and $8 \mathrm{~m}$ long; the ceiling is $3 \mathrm{~m}$ high and the shoulder height is $2 \mathrm{~m}$. Jujube trees are controlled below $2.2 \mathrm{~m}$ by pruning. After the shed is covered with film, pest control, fertilizer, and tree management were routinely conducted. The first sampling was performed on the second day of the buckle shed, with an interval of 14 days until the end of sampling. At the time of sampling, 3-5 branches of the middle and upper parts of the periphery of the crown of the jujube tree were sampled. Place the ice packs in the cling film and bring them back to the laboratory. The buds were removed with a blade and placed in a $-25{ }^{\circ} \mathrm{C}$ refrigerator for testing.

Methods. The activity of superoxide dismutase (SOD) was determined by the method of nitrogen blue tetrazolium (NBT) method [6], the activity of peroxidase (POD) was measured by guaiacol method [6], and the content of $\mathrm{O}_{2}{ }^{-}$. was determined by the method of Bi Lei [7]. The malonyldialdehyde (MDA) content was determined by Bi Lei's method [8].

\section{Results and Discussion}

Comparison of SOD activity under different treatments. From December 6 th to March 13 th (Fig.1), SOD has been on the rise throughout the process. The increase in shed cultivation is significantly greater than that in field cultivation. Shedding treatment improves the activity of SOD. In all treatments, the effect in shed 2 is better than others. Qiao Yongxu [9]'s research on lily bulbs showed that SOD declined firstly and then rose up. This change trend is inconsistent with this study, probably due to the different cold resistance of experimental materials. The experiment also showed that during the whole dormancy process, the SOD of the sprout shoots of Z. jujuba 'Zhanshanmizao' was maintained at a low level, which was in agreement with the results of Bi Lei [8] and Min zhuo [10]. The reason may be that the continuous low temperature in winter inhibited the activity of SOD enzyme.

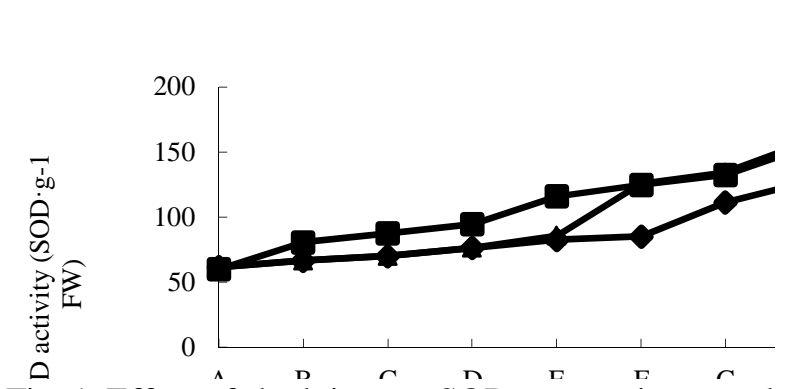

Fig. 1 Effect of shed time on SOD content in natural dormancy of Z. jujuba 'Zhanshanmizao'

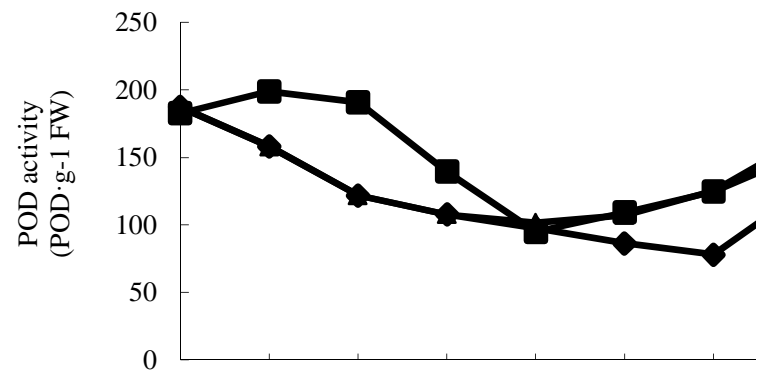

Fig. 2 Effect of shed time on POD content in natural dormancy of Z. jujuba 'Zhanshanmizao'

Note: A. December 6 th, 2016, B. December 20 th, 2016, C. December 27 th, 2016, D. January 3 th, 2017, E. January 17 th, 2017, F. January 31 st, 2017 , G. February 14 th, 2017, H. February 28 th, 2017, I. March 13 th. The same below.

Comparison of POD activity under different treatments. From December 6 th to March 13 th (Fig.2), the general trend of the activity of POD fell firstly and then rose up. During the dormancy period, the activity of POD in shoots with buds decreased, and after the dormancy ended, the temperature increased, the stress conditions were relieved, and the POD content increased rapidly. 
There was no significant difference between the two treatments, but the activity of POD increased compared to the cultivation of fields. Bi Lei [8], Zhao Hailiang [11] and other studies have shown that POD showed a downward trend during the dormancy of plants, and showed an increase after the end of dormancy. The results of this study are consistent with this. In this study, the trend of POD activity in Z. jujuba 'Zhanshanmizao' was similar to that of $\mathrm{O}_{2}{ }^{-}$. It showed that under the low temperature stress, the contents of $\mathrm{O}_{2}{ }^{-}$and $\mathrm{H}_{2} \mathrm{O}_{2}$ in the branches increased, which stimulated the synthesis of POD enzyme in the organism.

Comparison of $\mathrm{O}_{2}^{-} \cdot$ contents under different treatments. $\mathrm{O}_{2}^{-} \cdot$ can be decomposed by POD and SOD in plants to reduce its toxic effects on plants. From December 6 th to March 13 th (Fig.3), the total content of $\mathrm{O}_{2}^{-} \cdot$ in shoots of $Z$. jujuba 'Zhanshanmizao' showed a decreasing trend. There was no significant difference in the two treatments of shed cultivation, but compared with field cultivation, the activity of $\mathrm{O}_{2}^{-} \cdot$ was greatly reduced. $\mathrm{O}_{2}^{-}$and MDA have similar trends in the whole process. It has been showing a downward trend, which is consistent with the findings of Zhang Aung [12], Chen Xinhua [13], and Chen Dengwen [14]. The experiment results also show that $\mathrm{O}_{2}^{-} \cdot$ has been kept at a relatively low level, which may be due to the effect of SOD and POD, resulting in the removal of $\mathrm{O}_{2}^{-}$.

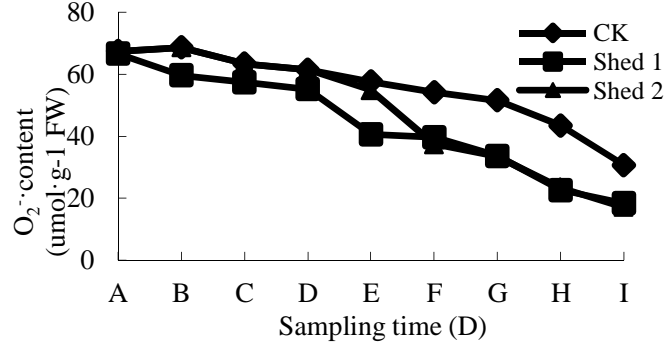

Fig. 3 Effect of shed time on $\mathrm{O}_{2}^{-} \cdot$ content in natural dormancy of Z. jujuba 'Zhanshanmizao'

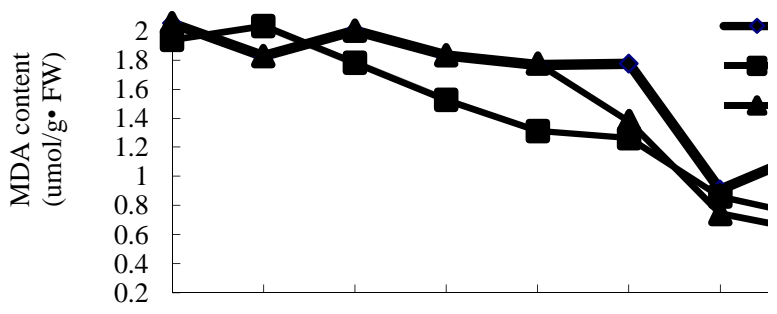

Fig.4 Effect of shed time on MDA content in natural dormancy of Z. jujuba 'Zhanshanmizao'

Comparison of MDA content under different treatments. The MDA content of Z. jujuba 'Zhanshanmizao' was increased in adversity. In the whole process (Fig.4), the total content of MDA in the shoots and branches of Z. jujuba 'Zhanshanmizao' showed a downward trend. The three trends are similar, from December 6 th to December 20 th as an upward trend, it may be at the deepest dormancy at this time, and the resistance ability of $Z$. jujuba was weakest in cold weather, and then decreased rapidly with the release of dormancy. There was no significant difference in the two treatments of shed cultivation, but the activity of MDA was greatly reduced compared with field cultivation. The content of MDA in the shoots of the jujube tree with bud sheds was significantly lower than that of the control, which was consistent with the results of Gao Jingcao [15]. The degree of membrane lipid peroxidation of the jujube branches under cold conditions was high, severely affected by freezing.

\section{Conclusions}

It was generally believed that the activation of the antioxidant system was the cause or condition for breaking the dormancy, that was, the oxygen free radicals were cleared by the activated antioxidative system, so that the activity of SOD, POD, CAT and other enzymes increased. Bud dormancy was related to the ability to scavenge free radicals. Superoxide dismutase SOD converts superoxide anion radical $\mathrm{O}_{2}{ }^{-}$. to $\mathrm{H}_{2} \mathrm{O}_{2}$ [16]. During the natural dormancy of $Z$. jujuba 'Zhanshanmizao', shed treatment could play a certain role in relieving dormancy of Z. jujuba 'Zhanshanmizao', and was also theoretically confirmed. In January 16 th, 2017, the effect of shed was the best, which sprouted 32 days ahead of field cultivation, and the price of fruit was four times that of normal, which increased the income of jujube farmers. The treatment of the sheds had a great effect on the release of the dormancy of Z. jujuba 'Zhanshanmizao'. If measures such as applying pesticides were applied on the basis of the shed, there may be better results. Look forward to breaking the dormancy in a better way to improve the economic value of fruit trees. 


\section{Acknowledgements}

This work financially supported by the double subject construction plan of Sichuan Agricultural University and special action plan project of science and technology rich people and strong county of Sichuan Province "Integration and demonstration of key technologies for industrialized development Z. jujuba 'Zhanshangmizao' of Santai County”.

\section{References}

[1] M.J. Liu: Jujube Quality Production Technical Manual (Beijing: China Agricultural Press, Beijing 2004).

[2] Y.Q. Zhu, X. Yang and T. Zhang: Journal of Sichuan Normal University (Natural Science Edition) Vol. 37 (2016), p. 467.

[3] G.Q. Liu, P.H. Zheng and Y.W. Teng: Journal of Fruit Science Vol. 5 (2012), p. 911.

[4] S.R. Xia: One Year Multi Ripening and High Yield Technology of Fresh Jujube (Golden Shield Press, Beijing 2008).

[5] M.X. Gao, Z.G. Yao, M.H. Bao and E.F. Ma: Chinese fruit tree Vol. 6 (2013), p. 15.

[6] Q.E. Xiong: Plant Physiology Experiment Course (Sichuan Science and Technology Press, Chengdu 2003).

[7] L. Bi: Changes in Substance and Physiological Metabolism during the Dormancy of Pear in July (thesis of master degree, Agricultural University of Hebei, Baoding, China 2009).

[8] L. Bi: Study on Dormancy Characteristics and Dormancy Release of Pear (thesis of master degree, Agricultural University of Hebei, Baoding, China 2006).

[9] Y.X. Qiao, Y.Y. Zhang, C. Chen and M.M. Chen: Southwest China Journal of Agricultural Sciences Vol. 23 (2010), p. 161.

[10] Z. Min: Expression of VvArp and VvCdk Genes Related to Grape Bud Dormancy and Changes of Nitrogen and Antioxidant Indexes during Dormancy Release (thesis of master degree, Northwest A\&F University, Xian, China 2015).

[11] G.Z. Wei and H.L. Zhao: Agricultural Science \& Technology Vol. 3 (2014), p. 367.

[12] A. Zhang, Y.X. Zheng, T. Chen, W.G. Geng, Y.L. Fang and H. Wang: Chinese Journal of Northwest Botany Vol. 32 (2012), p. 2075.

[13] X.H. Chen, B.L. Guo, J. Zhao and J.X. Yang: Journal of Hebei Agricultural University Vol. 32 (2009), p. 37-40.

[14] D.W. Chen, F. Wang and A.Q. Gao: Acta Botanica Sinica Vol. 20 (2000), p. 212.

[15] J.C. Gao, H.X. Wang and X.X. Li: Northern Horticulture Vol. 23 (2010), p. 18.

[16] Z. Tan, L. Li, D.G. Li, X.D. Chen, J. Li and D.S. Gao: Journal of Applied and Environmental Biology Vol. 18 (2012), p. 838. 